\title{
Stronger Two-Observer All-Versus-Nothing Violation of Local Realism
}

\author{
Adán Cabelld* \\ Departamento de Física Aplicada II, Universidad de Sevilla, 41012 Sevilla, Spain
}

(Dated: August 17, 2018)

\begin{abstract}
We introduce a two-observer all-versus-nothing proof of Bell's theorem which reduces the number of required quantum predictions from 9 [A. Cabello, Phys. Rev. Lett. 87, 010403 (2001); Z.-B. Chen et al., Phys. Rev. Lett. 90, 160408 (2003)] to 4, provides a greater amount of evidence against local realism, reduces the detection efficiency requirements for a conclusive experimental test of Bell's theorem, and leads to a Bell's inequality which resembles Mermin's inequality for three observers [N. D. Mermin, Phys. Rev. Lett. 65, 1838 (1990)] but requires only two observers.

PACS numbers: 03.65.Ud, 03.67.Pp, 03.67.-a, 42.50.-p
\end{abstract}

The Greenberger-Horne-Zeilinger (GHZ) proof 1, 2] of Bell's theorem [3] provided a direct contradiction between the Einstein-Podolsky-Rosen (EPR) [4] local elements of reality (LERs) by considering 4 perfect correlations predicted by quantum mechanics. However, while Bell's theorem required only two spacelike separated observers, the GHZ proof required three. A two-observer all-versus-nothing (AVN) proof [5] based on 9 perfect correlations was introduced in Refs. [6, 7], then adapted for two-photon systems [8], and recently tested in the laboratory [9]. Despite requiring only two observers, this AVN proof has two disadvantages when compared to GHZ: it is more complex, in the sense that it requires a higher number of quantum predictions, and provides less evidence against local realism than GHZ [10]; thus a conclusive experiment based on the two-observer proof would require higher detection efficiencies than one based on GHZ. Both disadvantages are indeed related: in the two-observer proof, 8 out of these 9 perfect correlations (i.e., $89 \%$ ) can be explained by LERs; in GHZ, only 3 out of these 4 perfect correlations (i.e., only $75 \%$ ) can be explained by LERs.

Here we introduce an AVN proof requiring two observers and only 4 perfect correlations. We then show that conclusive experiments based on this new proof would require lower detection efficiencies than those needed for the previous two-observer AVN proof, and derive the corresponding Bell inequality for real experiments.

Let us consider a two-photon system entangled both in polarization and path degrees of freedom [8, 9, 11] prepared in the state

$$
\begin{aligned}
|\psi\rangle= & \frac{1}{2}\left(|H u\rangle_{1}|H u\rangle_{2}+|H d\rangle_{1}|H d\rangle_{2}\right. \\
& \left.+|V u\rangle_{1}|V u\rangle_{2}-|V d\rangle_{1}|V d\rangle_{2}\right),
\end{aligned}
$$

where $|H\rangle_{j}$ and $|V\rangle_{j}$ represent horizontal and vertical polarization, and $|u\rangle_{j}$ and $|d\rangle_{j}$ denote two orthonormal path states for photon $j$. The state (1) can be viewed as a two-photon version of the four-qubit cluster state [12]. $\mathrm{Lu}$ [13] and $\mathrm{Lu}$ [14] have described procedures for preparing state (11).
Let us also consider 6 local observables on photon $j: 3$ for polarization degrees of freedom, defined by the operators

$$
\begin{aligned}
X_{j} & =|H\rangle_{j}\langle V|+| V\rangle_{j}\langle H|, \\
Y_{j} & =i\left(|V\rangle_{j}\langle H|-| H\rangle_{j}\langle V|\right), \\
Z_{j} & =|H\rangle_{j}\langle H|-| V\rangle_{j}\langle V|,
\end{aligned}
$$

and 3 for path degrees of freedom, defined by the operators

$$
\begin{aligned}
x_{j} & =|u\rangle_{j}\langle d|+| d\rangle_{j}\langle u|, \\
y_{j} & =i\left(|d\rangle_{j}\langle u|-| u\rangle_{j}\langle d|\right), \\
z_{j} & =|u\rangle_{j}\langle u|-| d\rangle_{j}\langle d| .
\end{aligned}
$$

Each of these observables can have only one of two possible values: -1 or 1 .

The proof has two steps. First, we will show that the 7 local observables $X_{1}, Y_{1}, x_{1}, X_{2}, Y_{2}, y_{2}$, and $z_{2}$ satisfy the EPR condition for LER; namely, "if, without in any way disturbing a system, we can predict with certainty (i.e., with probability equal to unity) the value of a physical quantity, then there exists an element of physical reality corresponding to this physical quantity" [4]. The observables $X_{1}, Y_{1}$, and $x_{1}$ of photon 1 are LERs because their values can be predicted with certainty from spacelike separated measurements on photon 2. Spacelike separation guarantees that photon 1 has not been disturbed in any way 15]. These predictions with certainty follow from the fact that state (1) satisfies the following equations:

$$
\begin{aligned}
X_{1} X_{2} z_{2}|\psi\rangle & =|\psi\rangle, \\
Y_{1} Y_{2} z_{2}|\psi\rangle & =-|\psi\rangle, \\
x_{1} Z_{2} x_{2}|\psi\rangle & =|\psi\rangle .
\end{aligned}
$$

Equation (8) tells us that, from the results of measuring $X_{2}$ and $z_{2}$ on photon 2 , we can predict with certainty the result $v\left(X_{1}\right)$ of measuring $X_{1}$ on photon 1. Equation (9) tells us that, from the results of measuring $Y_{2}$ and $z_{2}$ on photon 2 , we can predict with certainty the result $v\left(Y_{1}\right)$ of measuring $Y_{1}$ on photon 1. Equation (10) tells us that, 
from the results of measuring $Z_{2}$ and $x_{2}$ on photon 2, we can predict with certainty the result $v\left(x_{1}\right)$ of measuring $x_{1}$ on photon 1 .

Analogously, the observables $X_{2}, Y_{2}, y_{2}$, and $z_{2}$ of photon 2 are also LERs because state (11) also satisfies the following equations:

$$
\begin{aligned}
X_{1} z_{1} X_{2}|\psi\rangle & =|\psi\rangle, \\
Y_{1} z_{1} Y_{2}|\psi\rangle & =-|\psi\rangle, \\
Z_{1} y_{1} y_{2}|\psi\rangle & =-|\psi\rangle, \\
z_{1} z_{2}|\psi\rangle & =|\psi\rangle .
\end{aligned}
$$

Therefore, the results $v\left(X_{2}\right), v\left(Y_{2}\right), v\left(y_{2}\right)$, and $v\left(z_{2}\right)$ of measuring $X_{2}, Y_{2}, y_{2}$, and $z_{2}$ on photon 2 can be predicted with certainty from spacelike separated measurements on photon 1 .

Moreover, we can prove that two observables on the same photon, but corresponding to different degrees of freedom, like $X_{2}$ and $z_{2}$, are independent LERs in the sense that measuring one of them does not change the value of the other (thus there is no need for any additional assumptions beyond the EPR condition; see 16. for a similar discussion). For instance, a suitable measurement of $X_{2}$ does not change $v\left(z_{2}\right)$ because $v\left(z_{2}\right)$ can be predicted with certainty from a spacelike separated measurement on photon 1 [see Eq. [14] ], and this prediction is not affected by whether $X_{2}$ is measured before measuring $z_{2}$, or $X_{2}$ and $z_{2}$ are jointly measured. Therefore, the EPR criterion is enough to guarantee that $z_{2}$ has a value $v\left(z_{2}\right)$, which does not change by measuring $X_{2}$. A similar reasoning applies to all 7 local observables involved in the proof.

The second step of the proof consists of showing the contradiction between the predictions of quantum mechanics and those of local realistic theories. For this purpose, note that state (11) also satisfies the following equations:

$$
\begin{aligned}
X_{1} x_{1} Y_{2} y_{2}|\psi\rangle & =|\psi\rangle, \\
Y_{1} x_{1} X_{2} y_{2}|\psi\rangle & =|\psi\rangle .
\end{aligned}
$$

To be consistent with Eqs. (8), (9), (15), and (16), local realistic theories predict the following relations between the values of the LERs:

$$
\begin{aligned}
v\left(X_{1}\right) & =v\left(X_{2}\right) v\left(z_{2}\right), \\
v\left(Y_{1}\right) & =-v\left(Y_{2}\right) v\left(z_{2}\right), \\
v\left(X_{1}\right) v\left(x_{1}\right) & =v\left(Y_{2}\right) v\left(y_{2}\right), \\
v\left(Y_{1}\right) v\left(x_{1}\right) & =v\left(X_{2}\right) v\left(y_{2}\right),
\end{aligned}
$$

respectively. However, it is impossible to assign the values -1 or 1 in a way consistent with all Eqs. (17)-20). This can be proved as follows: In Eqs. (17)-20 every value appears twice; therefore, the product of Eqs. (17)(20) gives $1=-1$. We therefore conclude that the 4 predictions of quantum mechanics given by Eqs. (8), (9), (15), and (16) cannot be reproduced by EPR LERs.
The remarkable property of this AVN proof is that the contradiction appears after considering only 4 quantum predictions, while the previous two-observer AVN proof [6, 7, 8, 9] required 9 quantum predictions. Moreover, while a local realistic model can reproduce 8 out of the 9 predictions of the previous two-observer AVN proof, it can be easily seen that it can reproduce only 3 out of the 4 predictions in the proof presented here. In more practical terms, this means that an experimental realization of the previous AVN proof [9] requires photodetectors of a higher efficiency to avoid the detection loophole 17] than an experimental realization of the proposed proof. To show this, we will estimate the detector efficiency required for both proofs. For this purpose, it is useful to see both proofs as games [18].

Let us start by translating the new proof into a game in which a quantum-based strategy beats any classical strategy. Consider a team of two players, Alice and Bob, each of them isolated in a booth. Alice is asked one out of two possible questions: (I) "What are $v\left(X_{1}\right)$ and $v\left(x_{1}\right)$ ?" or (II) "What are $v\left(Y_{1}\right)$ and $v\left(x_{1}\right)$ ?" Bob is asked one out of 4 possible questions: (i) "What are $v\left(X_{2}\right)$ and $v\left(y_{2}\right)$ ?," (ii) "What are $v\left(X_{2}\right)$ and $v\left(z_{2}\right)$ ?," (iii) "What are $v\left(Y_{2}\right)$ and $v\left(y_{2}\right)$ ?," or (iv) "What are $v\left(Y_{2}\right)$ and $v\left(z_{2}\right)$ ?" Each of them must give one of the following answers: " -1 and -1 ," " -1 and 1," " 1 and -1 ," or " 1 and 1." When Alice is asked (I), Bob is asked (ii) or (iii); when Alice is asked (II), Bob is asked (i) or (iv). Since $v\left(X_{2}\right)$ represents a LER, Bob's answer to $v\left(X_{2}\right)$ must be independent on whether $v\left(X_{2}\right)$ is asked together with $v\left(y_{2}\right)$ or with $v\left(z_{2}\right)$. The same applies for Bob's answers to $v\left(Y_{2}\right), v\left(y_{2}\right)$, and $v\left(z_{2}\right)$. The team wins if their answers satisfy the corresponding equation of the set (17)-(20).

Assuming that the 4 possible combinations of questions are asked with the same frequency, no classical strategy allows the players to win in more than $3 / 4$ of the rounds. For instance, a simple optimal classical strategy is that each player always answers 1 to any question. The hidden set of local instructions

$$
\left\{\begin{array}{c|cc}
v\left(X_{1}\right) & v\left(X_{2}\right) & v\left(z_{2}\right) \\
v\left(Y_{1}\right) & v\left(Y_{2}\right) & v\left(y_{2}\right)
\end{array}\right\}
$$

where the vertical bar separates Alice's instructions from Bob's, is then

$$
G:=\left\{\begin{array}{l|ll}
1 & 1 & 1 \\
1 & 1 & 1
\end{array}\right\}
$$

This strategy only fails whenever Bob is asked (iv) (i.e., in $1 / 4$ of the rounds). However, there is a quantum strategy that never fails: the players can win all the rounds if they share pairs of photons in the state (10), and give as answers the results of the corresponding measurements on their photons.

In a real experiment for testing Bell's theorem, the low efficiency of detectors opens the possibility that nondetections could correspond to local instructions such as 
"if $X$ is measured, then the photon will not activate the detector." This allows local realistic theories to simulate the observed results. To estimate the detection efficiency required to experimentally discard these theories, let us introduce a modification to the rules of the game. Let us allow each player to give no answer whatsoever in a fraction $1-\eta$ of the rounds. If any of the players does not answer, then that round is not valid. This modification opens the possibility of the players also using a fraction of sets of local instructions like

$$
B_{1}:=\left\{\begin{array}{l|ll}
1 & 1 & 1 \\
0 & 1 & 1
\end{array}\right\}
$$

or

$$
B_{2}:=\left\{\begin{array}{l|ll}
1 & 1 & 1 \\
1 & 0 & 1
\end{array}\right\},
$$

where 0 means that the corresponding player will not answer the corresponding question. For instance, if the players share $B_{1}$, Alice will not answer question (II); and if they share $B_{2}$, Bob will not answer questions (iii) and (iv).

Let us suppose that the players are using sets of predefined answers (or, equivalently, that the observed data can be described by a local realistic theory). For instance, sets like $G$ with a frequency $1-p$, sets like $B_{1}$ with a frequency $p / 2$, and sets like $B_{2}$ with a frequency $p / 2$, where $p$ depends on the efficiency of the photodetector corresponding to photon $j$,

$$
\eta_{j}=1-p+\frac{p}{2} f_{j}+\frac{p}{2},
$$

where $f_{1}\left(f_{2}\right)$ is the probability that Alice (Bob) answers [i.e., she (he) does not get the instruction 0 in her (his) set] when they are using a $B_{1}\left(B_{2}\right)$ set. In our case $f_{j}=1 / 2$.

Let us calculate the minimum detection efficiency required to discard the possibility that the players are using this particular set of predefined answers (or, equivalently, that the observed data can be described by a local realistic theory). Then, to simulate the quantum probability of winning the game, the minimum value of $p$ is obtained by solving the equation

$$
P_{Q}=(1-p) P_{G}+\frac{p}{2} P_{B_{1}}+\frac{p}{2} P_{B_{2}},
$$

where $P_{Q}$ is the quantum probability of winning the game, $P_{G}$ is the probability of winning the game when the players use a $G$ set, and $P_{B_{j}}$ is the probability of winning when the players use a $B_{j}$ set and both answer the questions. In our case, $P_{Q}=1, P_{G}=3 / 4$ and $P_{B_{j}}=1$. Introducing these values in Eqs. (25) and (26), we arrive at the conclusion that our local model can simulate the quantum predictions if $\eta_{j} \leq 3 / 4=0.75$. An exhaustive examination of all possible sets of local instructions shows that the previously presented model is indeed optimal and therefore we conclude that local realistic theories cannot simulate the quantum predictions if

$$
\eta_{j}>3 / 4
$$

which is the same efficiency needed for a loophole-free experiment based on the three-observer version of GHZ's proof [19]. Indeed, the efficiency required for a twoobserver AVN proof based on the state (11) can be lowered to $\eta_{j}>11 / 16 \approx 0.69$ if we consider 12 quantum predictions 20 .

Let us compare these efficiencies with that required for a loophole-free experiment based on the two-photon version [8, 9] of the the two-observer AVN proof [6, 7]. To be consistent with 9 specific predictions of quantum mechanics (for details, see []]), local realistic theories predict the following 9 relations between the values of the LERs:

$$
\begin{aligned}
v\left(Z_{1}\right) & =-v\left(Z_{2}\right), \\
v\left(z_{1}\right) & =-v\left(z_{2}\right), \\
v\left(X_{1}\right) & =-v\left(X_{2}\right), \\
v\left(x_{1}\right) & =-v\left(x_{2}\right), \\
v\left(Z_{1} z_{1}\right) & =v\left(Z_{2}\right) v\left(z_{2}\right), \\
v\left(X_{1} x_{1}\right) & =v\left(X_{2}\right) v\left(x_{2}\right), \\
v\left(Z_{1}\right) v\left(x_{1}\right) & =v\left(Z_{2} x_{2}\right), \\
v\left(X_{1}\right) v\left(z_{1}\right) & =v\left(X_{2} z_{2}\right), \\
v\left(Z_{1} z_{1}\right) v\left(X_{1} x_{1}\right) & =-v\left(Z_{2} x_{2}\right) v\left(X_{2} z_{2}\right) .
\end{aligned}
$$

It is impossible to assign the values -1 or 1 in a way consistent with all Eqs. (28)-(36) 7]. To calculate the detection efficiency required for a conclusive test based on this proof we will convert this impossibility into a game in which a quantum-based strategy beats any classical strategy. Consider again a team of two players, each of them isolated in a booth. Alice is asked one out of three possible questions: (I) "What are $v\left(Z_{1}\right)$ and $v\left(x_{1}\right)$ ?," (II) "What are $v\left(X_{1}\right)$ and $v\left(z_{1}\right)$ ?," or (III) "What are $v\left(Z_{1} z_{1}\right)$ and $v\left(X_{1} x_{1}\right)$ ?" Analogously, Bob is asked one out of three possible questions: (i) "What are $v\left(Z_{2}\right)$ and $v\left(z_{2}\right)$ ?," (ii) "What are $v\left(X_{2}\right)$ and $v\left(x_{2}\right)$ ?," or (iii) "What are $v\left(Z_{2} x_{2}\right)$ and $v\left(X_{2} z_{2}\right)$ ?" Each of them must give one of the following answers: "-1 and $-1, "$ " -1 and $1, "$ "1 and $-1, "$ or " 1 and 1." An interesting feature of this game is that it does not require a promise: all nine possible combinations of questions are legitimate. Assuming that all questions are asked with the same frequency, no classical strategy allows the players to win in more than $8 / 9$ of the rounds. However, the players can win all the rounds using a quantum strategy [7]. An optimal classical strategy consists on using sets of instructions

$$
\left\{\begin{array}{cc|cc}
v\left(Z_{1}\right) & v\left(z_{1}\right) & v\left(Z_{2}\right) & v\left(z_{2}\right) \\
v\left(X_{1}\right) & v\left(x_{1}\right) & v\left(X_{2}\right) & v\left(x_{2}\right) \\
v\left(Z_{1} z_{1}\right) & v\left(X_{1} x_{1}\right) & v\left(Z_{2} x_{2}\right) & v\left(X_{2} z_{2}\right)
\end{array}\right\}
$$


like

$$
G:=\left\{\begin{array}{rr|rr}
1 & 1 & -1 & -1 \\
1 & 1 & -1 & -1 \\
1 & 1 & 1 & 1
\end{array}\right\},
$$

which gives correct answers except when Alice is asked (III) and Bob is asked (iii).

Now let us suppose that each player is also allowed to give no answer in a fraction $1-\eta$ of the rounds. This is equivalent to assuming that they can use sets of local instructions like

$$
B_{1}:=\left\{\begin{array}{rr|rr}
1 & 1 & -1 & -1 \\
1 & 1 & -1 & -1 \\
0 & 0 & 1 & 1
\end{array}\right\},
$$

or

$$
B_{2}:=\left\{\begin{array}{rr|rr}
1 & 1 & -1 & -1 \\
1 & 1 & -1 & -1 \\
1 & 1 & 0 & 0
\end{array}\right\}
$$

In this case, an optimal ensemble of sets of local instructions turns out to be $G$ sets with frequency $1-p, B_{1}$ sets with frequency $p / 2$, and $B_{2}$ sets with frequency $p / 2$. Therefore, $P_{Q}=1, P_{G}=8 / 9, P_{B_{j}}=1$, and $f_{j}=2 / 3$, which leads us to conclude that, to avoid the detection loophole in the two-observer AVN proof in [6, 7, [] $]$, we need

$$
\eta_{j}>5 / 6 \approx 0.83,
$$

which is higher than the value, given by inequality (27), required for the two-observer AVN proof introduced in this Letter.

AVN proofs follow directly from perfect correlations predicted by quantum mechanics. However, in a laboratory realization of the experiment, the observed correlations will not be as perfect as the proof requires. It is therefore convenient to derive Bell's inequalities whose validity is necessary for the observed correlations to be consistent with a very general probabilistic local realistic theory, which are violated by the quantum predictions by an amount allowing significant room for the blurring effect of the imperfections in real experiments. The relevant features of the AVN proof derive from the fact that, for the state (11),

$$
\left\langle\psi\left|X_{1} X_{2} z_{2}-Y_{1} Y_{2} z_{2}+X_{1} x_{1} Y_{2} y_{2}+Y_{1} x_{1} X_{2} y_{2}\right| \psi\right\rangle=4
$$

while, as can be easily checked, in any local realistic theory, this expected value must satisfy

$$
\left|\left\langle X_{1} X_{2} z_{2}-Y_{1} Y_{2} z_{2}+X_{1} x_{1} Y_{2} y_{2}+Y_{1} x_{1} X_{2} y_{2}\right\rangle\right| \leq 2 .
$$

Inequality (43) resembles Mermin's inequality for three observers [5], in the sense that quantum mechanics predicts a violation of 4 , which is indeed the maximum possible violation of inequality (43), while the local realistic limit is 2. However, inequality (43) requires only two observers, as in the original Bell inequality [3] .

Summing up, we have introduced an AVN proof which combines the most interesting features appearing, separately, in previous AVN proofs: it is simple (the contradiction to EPR LERs follows from only 4 quantum predictions), provides a greater amount of evidence against local realism (only 3 out of these 4 predictions can be reproduced by LERs), and requires only two observers. In addition, we have shown that a conclusive experimental test of this proof would require lower efficiency detectors than those needed for the previous two-observer AVN proof, and we have derived the corresponding Bell inequality for real experiments, which resembles Mermin's inequality for three observers but requires only two observers.

The author thanks A. Broadbent, E. Galvão, A. Lamas-Linares, J.-Å. Larsson, C.-Y. Lu, S. Lu, E. Santos, and H. Weinfurter for useful comments, and acknowledges support by Projects No. BFM2002-02815 and No. FQM-239.

* Electronic address: adan@us.es

[1] D.M. Greenberger, M.A. Horne, and A. Zeilinger, in Bell's Theorem, Quantum Theory, and Conceptions of the Universe, edited by M. Kafatos (Kluwer Academic, Dordrecht, Holland, 1989), p. 69.

[2] N.D. Mermin, Phys. Rev. Lett. 65, 3373 (1990).

[3] J.S. Bell, Physics (Long Island City, NY) 1, 195 (1964).

[4] A. Einstein, B. Podolsky, and N. Rosen, Phys. Rev. 47, 777 (1935).

[5] The term "all versus nothing," to designate proofs based only on perfect correlations, was first used in N.D. Mermin, Phys. Rev. Lett. 65, 1838 (1990). Hardy's proof [L. Hardy, Phys. Rev. Lett. 71, 1665 (1993)] is not of this type.

[6] A. Cabello, Phys. Rev. Lett. 86, 1911 (2001).

[7] A. Cabello, Phys. Rev. Lett. 87, 010403 (2001).

[8] Z.-B. Chen, J.-W. Pan, Y.-D. Zhang, Č. Brukner, and A. Zeilinger, Phys. Rev. Lett. 90, 160408 (2003).

[9] T. Yang, Q. Zhang, J. Zhang, J. Yin, Z. Zhao, M. Żukowski, Z.-B. Chen, and J.-W. Pan, quant-ph/0502085 [Phys. Rev. Lett. (to be published)]; M. Barbieri, C. Cinelli, F. De Martini, and P. Mataloni, quant-ph/0505098

[10] W. van Dam, P. Grunwald, and R.D. Gill, quant-ph/0307125

[11] P.G. Kwiat, J. Mod. Opt. 44, 2173 (1997); P.G. Kwiat and H. Weinfurter, Phys. Rev. A 58, R2623 (1998).

[12] H.-J. Briegel and R. Raussendorf, Phys. Rev. Lett. 86, 910 (2001); R. Raussendorf and H.-J. Briegel, Phys. Rev. Lett. 86, 5188 (2001); P. Walther, K.J. Resch, T. Rudolph, E. Schenck, H. Weinfurter, V. Vedral, M. Aspelmeyer, and A. Zeilinger, Nature (London) 434, 169 (2005).

[13] C.-Y. Lu (private communication).

[14] S. Lu (private communication). 
15] A. Einstein, Dialectica 2, 320 (1948).

[16] L. Marinatto, Phys. Rev. Lett. 90, 258901 (2003); A. Cabello, Phys. Rev. Lett. 90, 258902 (2003).

[17] P.M. Pearle, Phys. Rev. D 2, 1418 (1970); E. Santos, Phys. Rev. A 46, 3646 (1992).

[18] L. Vaidman, Found. Phys. 29, 615 (1999); G. Brassard,
A. Broadbent, and A. Tapp, quant-ph/0407221

[19] J.-Å. Larsson, Phys. Rev. A 57, R3145 (1998); Phys. Rev. A 59, 4801 (1999).

[20] A. Cabello, Phys. Rev. A 72, 050101(R) (2005). 